\title{
A treatment planning inter-comparison of proton and intensity modulated photon radiotherapy
}

\author{
Antony J. Lomax ${ }^{\mathrm{a}, *}$, Thomas Bortfeld ${ }^{\mathrm{b}}$, Gudrun Goitein ${ }^{\mathrm{a}}$, Juergen Debus ${ }^{\mathrm{b}}$, Christine Dykstra ${ }^{\mathrm{c}}$, \\ Pierre-Alain Tercier ${ }^{\mathrm{d}}$, Philippe A. Coucke ${ }^{\mathrm{e}}$, Rene O. Mirimanoff ${ }^{\mathrm{e}}$ \\ ${ }^{a}$ Department of Radiation Medicine, The Paul Scherrer Institute, CH-5200, Villigen PSI, Switzerland \\ ${ }^{\mathrm{b}}$ Forschungsschwerpunkt Radiologie, Deutsches Krebsforschungszentrum, Heidelberg, Germany \\ ${ }^{\mathrm{c}}$ School of Computing Science, Simon Fraser University, Vancouver, Canada \\ ${ }^{\mathrm{d}}$ Institute of applied Radiophysics, Lausanne, Switzerland \\ ${ }^{\mathrm{e}}$ Department of Radio-oncology, University Hospital of Lausanne, CHUV, Lausanne, Switzerland
}

Received 13 May 1998; received in revised form 12 December 1998; accepted 14 January 1999

\begin{abstract}
Purpose: A comparative treatment planning study has been undertaken between standard photon delivery techniques, b intensity modulated photon methods and spot scanned protons in order to investigate the merits and limitations of each of these treatment approaches.

Methods: Plans for each modality were performed using CT scans and planning information for nine patients with varying indications and lesion sites and the results have been analysed using a variety of dose and volume based parameters.

Results: Over all cases, it is predicted that the use of protons could lead to a reduction of the total integral dose by a factor three compared to standard photon techniques and a factor two compared to IM photon plans. In addition, in all but one Organ at Risk (OAR) for one case, protons are predicted to reduce both mean OAR dose and the irradiated volume at the 50\% mean target dose level compared to both photon methods. However, when considering the volume of an OAR irradiated to $70 \%$ or more of the target dose, little difference could be shown between proton and intensity modulated photon plans. On comparing the magnitude of dose hot spots in OARs resulting from the proton and IM photon plans, more variation was observed, and the ranking of the plans was then found to be case and OAR dependent.

Conclusions: The use of protons has been found to reduce the medium to low dose load (below about 70\% of the target dose) to OARs and all non-target tissues compared to both standard and inversely planned photons, but that the use of intensity modulated photons can result in similar levels of high dose conformation to that afforded by protons. However, the introduction of inverse planning methods for protons is necessary before general conclusions on the relative efficacy of photons and protons can be drawn. C) 1999 Elsevier Science Ireland Ltd. All rights reserved.
\end{abstract}

Keywords: Proton therapy; Intensity modulated pholon radiotherapy; Comparative treatment planning

\section{Introduction}

Since the widespread introduction of computer tomographic (CT) scanners and other three-dimensional (3D) imaging modalities, treatment planning has become an increasingly important aspect of radiotherapy. In many ways, progress in planning technology has run in parallel with, and in some cases has been the driving force behind, many of the advances in delivery hardware that are now entering clinical use. These advances mainly have the aim of improving delivery methods such that the high dose volume approaches the volume of the clinical target, thus reducing unnecessary dosage to healthy tissues. Such highly

\footnotetext{
* Corresponding author.
}

conformal methods are now gaining acceptance in the clinical environment and in the near future are likely to become the routine treatment method for many indications.

Many of the proposed methods for improved localisation of the high dose volume centre either on the use of sophisticated planning and delivery methods and/or on the use of radiations which show improved dose localisation characteristics. In mega-voltage X-ray therapy, the use of asymmetric and dynamic wedges, irregularly shaped fields (i.e. using customised blocks or multi-leaf collimators) and the delivery of non-uniform intensity profiles of the incident radiation using compensators have all contributed to reducing the treated volume. In parallel with these developments, treatment planning systems have become correspondingly more elaborate, providing the radiation oncol- 
Table 1

List of all the cases, their indications and plan descriptions ${ }^{\mathrm{a}}$

\begin{tabular}{llll}
\hline Case number & Indication & Photons & IMRT photons \\
\hline 1 & Meningioma & LL,RL,LPO & 9 fields \\
2 & Malignant melanoma & A,LPO,RAO & 9 fields \\
3 & Klatskin tumour & LL,RAO,RPO & 9 fields \\
4 & Metastasising prostate carcinoma & A,LL,RL & 9 fields \\
5 & Cervix cancer & LAO,RAO,LPO,RPO & 9 fields \\
6 & Chromophobic pituitary adenoma & RL,LL,SPO & 9 fields \\
7 & Simulated thyroid carcinoma & A,RL,LL & 9 fields \\
8 & Acinus cellcarcinoma & - & A fields \\
9 & Relapsing medulloblastoma & - & LL,LAO,SALO fields \\
\hline
\end{tabular}

${ }^{a}$ For the photon and proton plans, the following nomenclature to describe field incidences has been adopted: L, lateral; O, oblique; L, left; R, right; A, anterior; P, posterior; $\mathrm{S}$, superior.

ogist with many options for conforming the dose in three dimensions.

With the number of degrees of freedom now available in the planning of radiation therapy, it is perhaps inevitable that great interest is being shown in the optimisation of treatment plans. In recent years, such techniques have been used in the calculation of beam weights $[30,35]$, for determination of wedge angles using dynamic wedges [42] or for the selection of beam directions $[5,16]$. However, perhaps the most promising of the optimisation techniques are that group which aim to modulate the cross-field fluence of the applied beams $[4,17,20,38,39,50]$.

One of the early attempts at reducing treatment volumes came with the use of protons in radiotherapy, as proposed by Wilson [51]. Wilson recognised the clear physical advantage of the proton depth-dose characteristic over that of a photon beam, and this was used as the rationale to treat patients with protons beginning around the early 1960s. By the end of 1997, over 24000 patients had been treated using protons at 21 sites world-wide, the majority at Massachusetts General Hospital using the original Harvard Cyclotron [44]. For the treatment of deep seated tumours with protons, all the currently operational facilities use the so called passive scattering method $[3,22,23]$ that was first developed at the Harvard Cyclotron. More recently, however, some groups have proposed the use of scanned particle beams for dynamic delivery [21,40], which can both improve dose conformation [48] and has the potential to deliver non-uniform fields in three-dimensions $[9,28]$.

A body of literature predicts that the use of protons in some cases can provide considerable improvements in dose conformation when compared to conventional X-ray delivery methods [24,31-34,45-47]. Such comparative treatment planning studies have commonly been used as a method of evaluating different treatment techniques and modalities (see, e.g. $[15,19,36]$ ) as well as in evaluating the usefulness of intensity modulated X-ray therapy in comparison with standard planning techniques and between different optimisation regimes [49]. We have previously reported on the results of a treatment planning intercomparison between protons and intensity modulated photons in a single case of childhood Medulloblastoma [34]. However, to date, detailed comparisons of proton therapy with state-of-theart intensity modulated X-ray techniques in a number of different sites and indications have not been performed. Here we present the results of a three-way treatment planning inter-comparison between standard photons, intensity modulated photons and protons as applied to a total of nine cases of varying indication and anatomical site.

\section{Materials and methods}

\subsection{Data collection and exchange}

Three centres have been involved in the study: the Paul Scherrer Institute (PSI); the University Hospital of Lausanne (CHUV) in Switzerland and the German Cancer Research Centre (DKFZ) in Heidelberg. Each centre provided three cases for evaluation, listed in full with their indications in Table 1. The conversion of planning data between the different planning systems was performed at PSI and all data transfers were made using FTP over internet connections. Volumes of interest (VOIs) were defined at the originating institute and both the CT and VOI files were distributed to the various centres for planning. All CT data were volumetric, with a maximum slice separation of $5 \mathrm{~mm}$, and VOIs were defined in 3D on all the relevant slices. For the sake of simplicity, the target volumes were, in all cases, assumed to include a safety margin, and were thus considered to be planning target volumes as defined in ICRU 50 [18]. Three dimensional dose distributions were calculated by each of the planning systems and were collected for evaluation at PSI using an in-house developed plan comparison package [26].

\subsection{Planning methods}

All standard photon plans were provided by either CHUV, using the DOSIGRAY planning system, or were planned using the VOXELPLAN system at either DKFZ or PSI [1,2]. By routinely available, we refer to the use of a limited number of beam ports, each of which can be indi- 


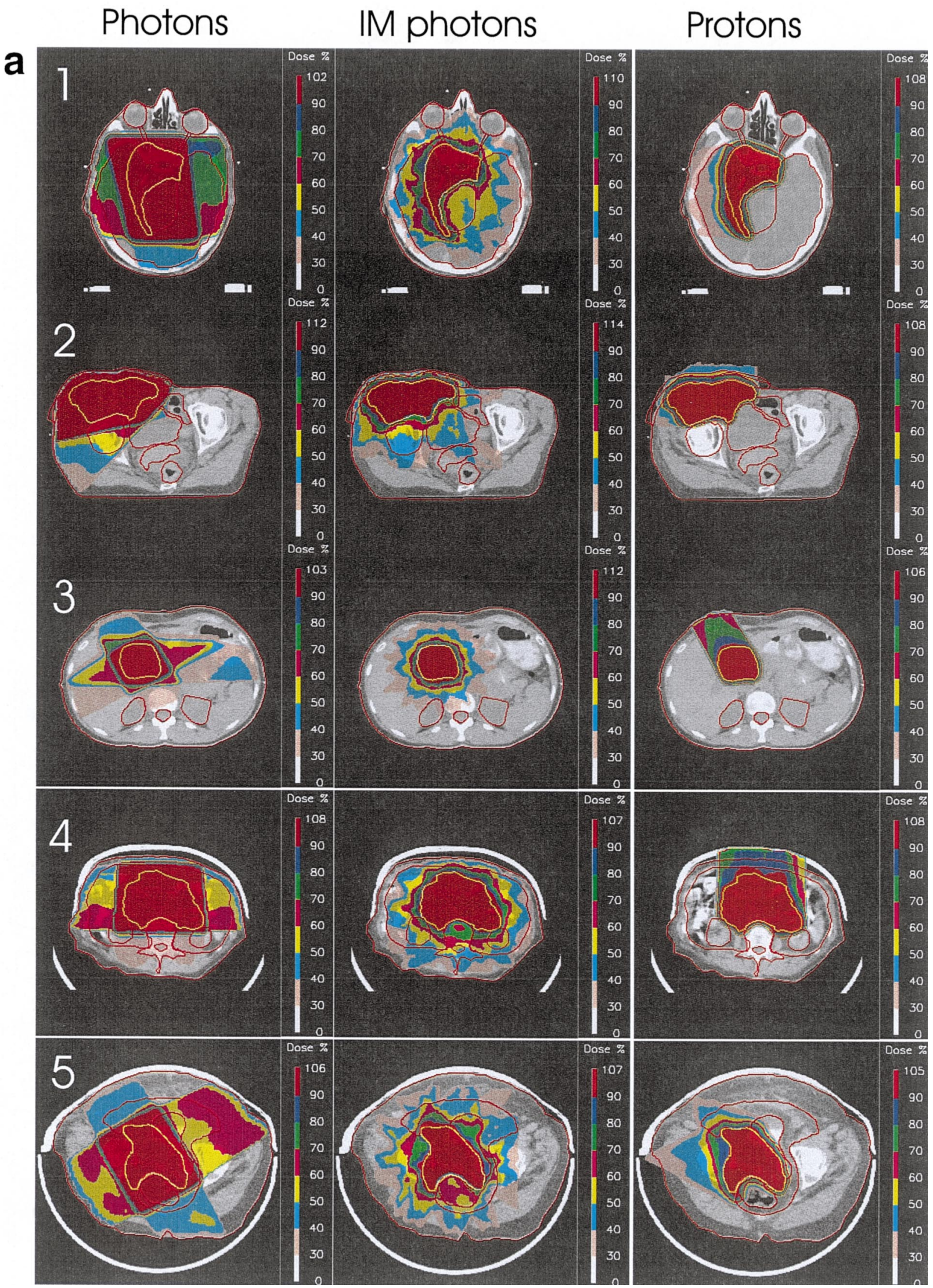

Fig. 1(a). 




Fig. 1. (a) Central slices from each plan for cases 1-5. (b) Central slices from each plan for cases 6-9. Dose levels are as indicated in the colour bars on the right hand side of each image. Note, for cases 8 and 9, no standard photon plans were performed.

vidually shaped but for which the beam intensity is fixed or is modified only by the use of fixed or dynamic wedges. Intensity modulated photon treatment plans were optimised using a forerunner of the KonRad inverse planning program developed at DKFZ $[4,8,41]$ which has been investigated dosimetrically [7] and is currently in clinical use at DKFZ, and, in a modified form, at the Memorial Sloan Kettering Cancer Centre [25]. It is based on an iterative steepest descent optimisation technique, which considers prescribed minimum and maximum dose constraints in the target as well as upper dose limits in organs at risk. DVHs constraints can also be defined [8], but this capability has not been used for the present investigation. In addition, socalled penalties are used to control the relative importance of these constraints. The dose is calculated using a simplified pencil beam algorithm in which the photon scatter kernel is assumed to be invariant with depth (using a simplified version of the technique described by Bortfeld [6]). 
Tissue inhomogeneities can be considered through onedimensional path length correction, but they are ignored in the present study, which uses an older implementation of the code. Although the inclusion of heterogeneity corrections would certainly alter the resultant IM matrices, we have assumed that the optimisation process would mostly compensate for these effects and we don't expect that the dose distributions would vary significantly from those presented in this paper. For all the cases studied here, IM photon plans were calculated using nine evenly spaced field directions (see Table 1).

All proton plans presented in this paper have been calculated using a treatment planning system developed at PSI specifically for the delivery of proton therapy using spot scanning [27,40,43]. Although the clinical advantages of this method over the more widespread passive scattering approach are a matter of debate [14,48], it nevertheless provides the most flexible method of particle delivery for conforming the dose in all three dimensions. Dose calculations are performed by taking into consideration all those Bragg peaks, deposited on a $5 \mathrm{~mm}$ matrix, which are inside or within $5 \mathrm{~mm}$ of the surface of the planning target volume. The relative weights of all these peaks are then calculated using a least squared minimisation technique [27]. However, in contrast to the photon technique, the optimisation for all the proton plans presented here have been performed for individual field directions only and with the single criteria of producing a relatively homogenous $( \pm 5$ $10 \%)$ dose across the target volume. Critical organs are ignored by the optimisation process and multiple field dose distributions were formed by the simple addition of the individually optimised fields. Thus, although optimised, the proton plans presented here are not IM plans in the commonly accepted sense of that term, and can more accurately be described as having been planned using conventional, forward planning techniques. For all the proton dose calculations, in-homogeneities along the beam direction were taken into account and lateral spread was modelled taking into account the effects of the initial phase space of the beam, nozzle-to-patient air gaps and multiple coulomb scattering within the patient.

\section{Results}

Example slices for each case are shown in Fig. 1a (cases 1-5) and Fig. 1b (cases 6-9). For each case, the standard photon plan is shown on the left, the intensity modulated photon plan in the middle and the proton plan on the right. Descriptions of each plan for each case are shown in Table 1 , and the normal tissue constraints of individual OARs used by the IM photon optimisation are shown in Table 4 . Note that for cases 8,9 , no standard photon plans have been performed. In all cases, the dose distributions have been normalised to the mean target dose delivered by each plan, and the doses have been coded using the colour band- ing indicated to the right hand side of each image. The banding levels (as a percentage of the normalisation dose) indicate the transition level from one colour band to the next and in all cases have been equally spaced between 30 and $90 \%$ of the normalisation dose. The maximum displayed dose value indicates the maximum dose anywhere within the distribution.

In this section, we will first present a dosimetric summary of all the cases, in the form of target coverage, integral dose estimates and doses to organs at risk (OAR) and follow this with more detailed studies of four cases of particular interest.

\subsection{General results}

\subsubsection{Target coverage and non-target tissue doses}

Tables 2 and 3 summarise some general results of the planning inter-comparisons. As a measure of the target coverage achieved by each planning method, we have calculated the minimum significant dose that the target receives from each plan. Within the target volume, we define this to be the maximum dose level for which a connected region, of defined volume, exists within which all dose grid points are irradiated to this dose level or below. Thus, minimum significant dose provides a measure of the magnitude of a cold spot of significant size within the target volume [11]. In this work, the defined volume has been taken to be equivalent to the volume of a sphere of radius $0.75 \mathrm{~cm}$ (the ICRU definition of a hot spot in an OAR [18]).

From the cold spot analysis shown in Table 2 it can be seen that the proton plans generally provide good coverage of the target, with only one proton plan predicting a cold spot lower than $95 \%$ (case 5 with a minimum significant dose of 93\%). For both the standard and IM photon plans, the results vary somewhat, with three standard photon plans (worst case 59\% cold spot for case 2) and five IM photon plans (worst case $77 \%$ for case 7 ) having cold spots of less than $95 \%$.

Table 3 provides estimates of the volume of non-target tissue irradiated to a dose level of $30 \%$ or more, which we use as a surrogate of the integral dose in order to summar-

Table 2

Minimum significant doses to the targets of each case as a percentage of the mean target dose (for full description, see text)

\begin{tabular}{llll}
\hline & \multicolumn{2}{l}{ Minimum significant dose in target $(\%)$} \\
\cline { 2 - 4 } Case & Proton & Photon & IM \\
\hline 1 & 96 & 100 & 91 \\
2 & 96 & 59 & 87 \\
3 & 95 & 97 & 97 \\
4 & 96 & 77 & 93 \\
5 & 93 & 95 & 81 \\
6 & 98 & 97 & 97 \\
7 & 94 & 76 & 77 \\
8 & 96 & - & 87 \\
9 & 95 & - & 93 \\
\hline
\end{tabular}


Table 3

The volume (in $\mathrm{cm}^{3}$ ) of non-target tissue that would receive $>30 \%$ of the prescribed dose ${ }^{\mathrm{a}}$

\begin{tabular}{lccr}
\hline & \multicolumn{2}{l}{ Volume $\left(\mathrm{cm}^{3}\right)$ receiving $>30 \%$ prescribed dose } \\
\cline { 2 - 4 } Case & Proton & Photon & \multicolumn{1}{c}{ IM } \\
\hline 1 & 216 & $847(3.9)$ & $369(1.7)$ \\
2 & 849 & $3348(3.9)$ & $3845(4.5)$ \\
3 & 324 & $1567(4.8)$ & $593(1.8)$ \\
4 & 2387 & $7759(3.2)$ & $6765(2.8)$ \\
5 & 1563 & $4403(2.8)$ & $3374(2.2)$ \\
6 & 366 & $1010(2.8)$ & $775(2.1)$ \\
7 & 1039 & $3475(3.3)$ & $2090(2.0)$ \\
8 & 792 & - & $977(1.2)$ \\
9 & 101 & - & $100(1.0)$ \\
Mean & - & 3.5 & 2.1 \\
\hline
\end{tabular}

${ }^{a}$ For the photon and intensity modulated plans, the volumes relative to the proton plans are shown in brackets. Over all cases, the mean $30 \%$ volumes relative to the proton plans are shown in the last row.

ise the dose delivered to all non-target tissues. In all cases, the photon and intensity modulated plans predict that a
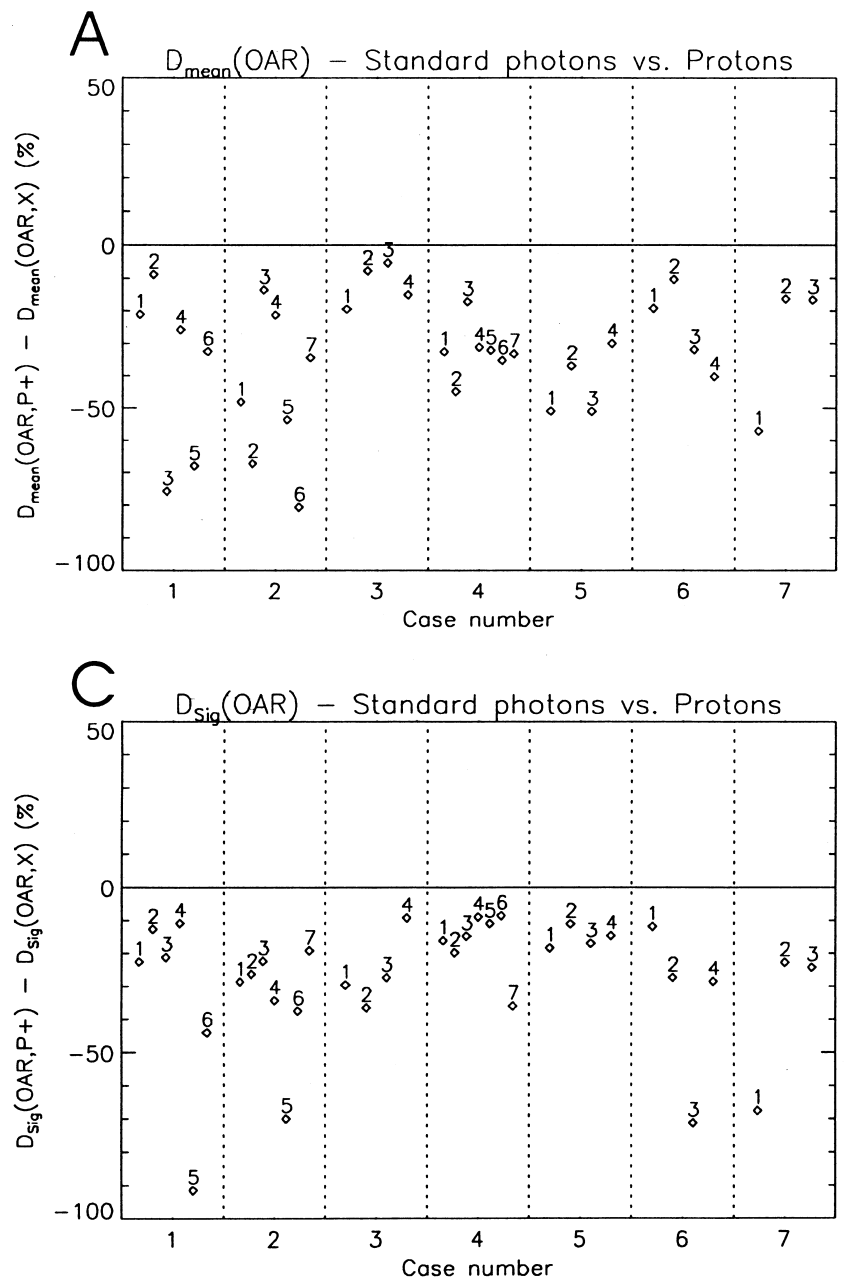

higher volume of normal tissue will be irradiated to the $30 \%$ dose level than for the corresponding proton plan, with this volume being 1.2-4.8 times higher for the standard photon plans and 1.0-4.5 times higher for the intensity modulated plans. The mean ratios over all cases for non-target tissue irradiation at the $30 \%$ level are 3.5 for the photon plans and 2.1 for the intensity modulated plans (Table 3).

\subsubsection{Organ at risk (OAR) doses}

The predicted doses to all organs at risk for all the cases are summarised in Figs. 2 and 3. Fig. 2A,B shows, respectively, the difference in the mean dose predicted for each OAR of each case between the proton plan and the corresponding standard photon plan (denoted as $D_{\text {mean }}(\mathrm{OAR}, \mathrm{P}+)-D_{\text {mean }}$ $(\mathrm{OAR}, \mathrm{X})$ ) and the intensity modulated photon plan (denoted as $\left.D_{\text {mean }}(\mathrm{OAR}, \mathrm{P}+)-D_{\text {mean }}(\mathrm{OAR}, \mathrm{IMX})\right)$. The individual OARs of each case are identified numerically according to the assignments given in Table 4 . As the differences between the proton and either photon plan is plotted, a dosimetric advantage in a particular OAR for the proton plan appears
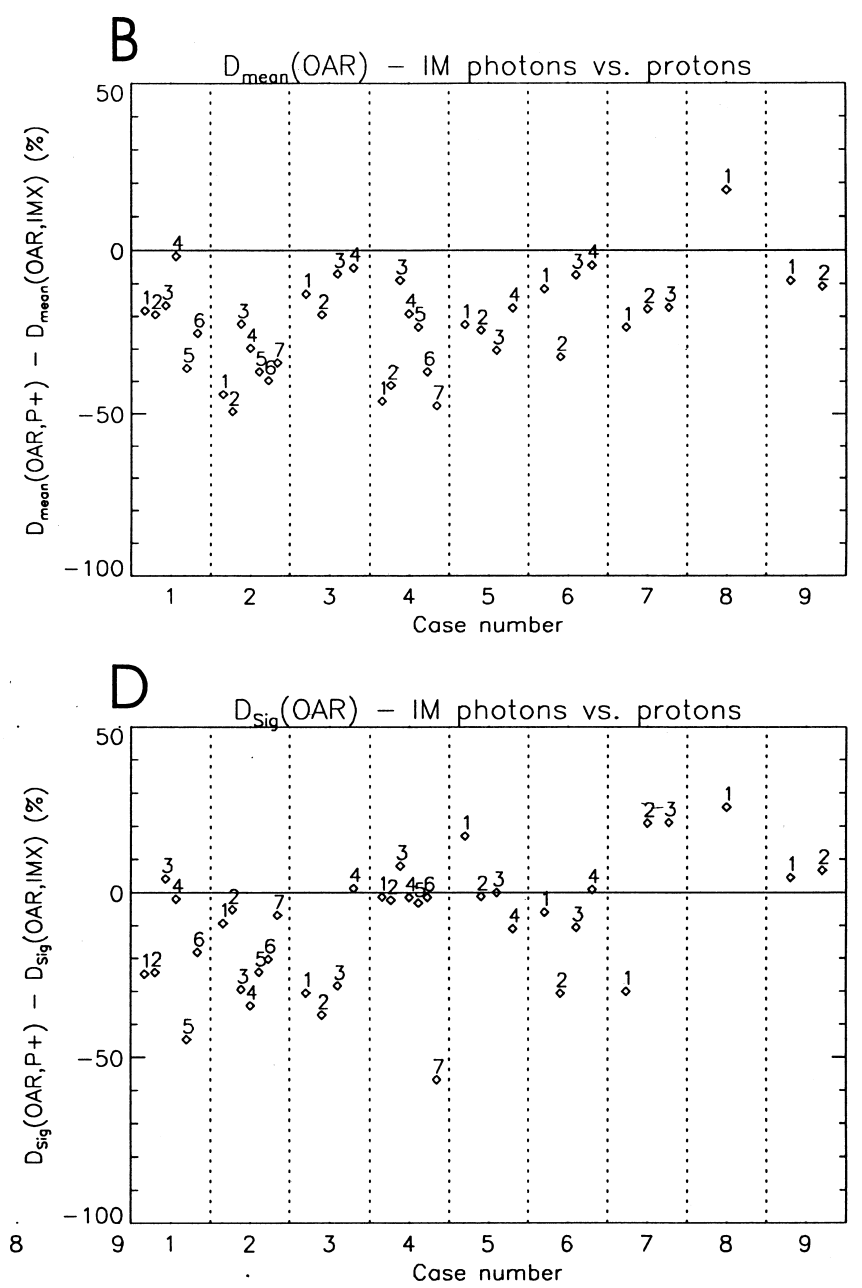

Fig. 2. Differences between proton plans and standard (A,C) and intensity modulated (B,D) plans for mean $\left(D_{\text {mean }}\right)$ and maximum significant dose $\left(D_{\text {sig }}\right)$, respectively. 

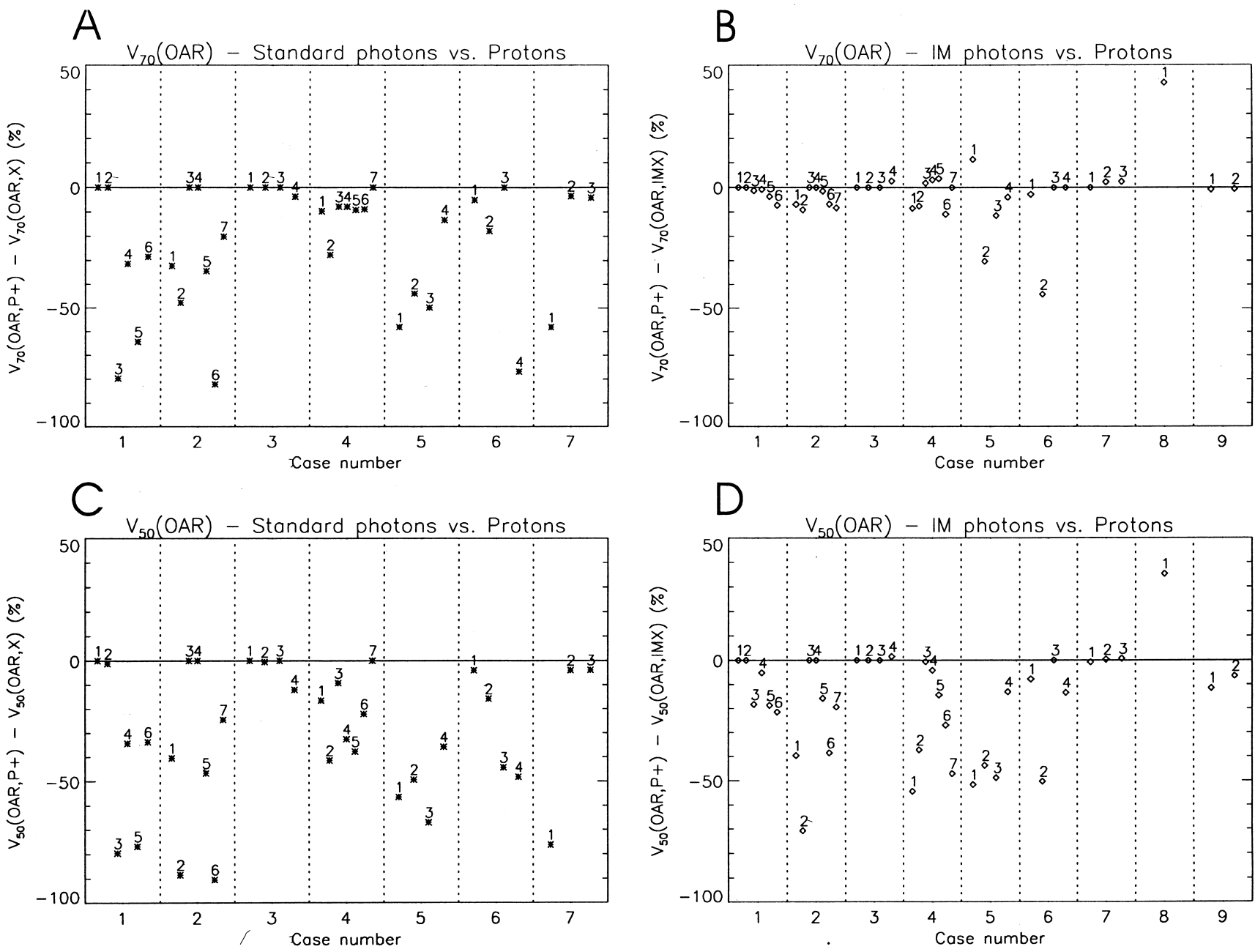

Fig. 3. Differences between proton plans and both standard (A,C) and intensity modulated (B,D) plans for the volumes of OARs irradiated to $50 \%$ or more $\left(V_{50}\right)$ and $70 \%$ or more $\left(V_{70}\right)$, respectively.

as a point below the zero line, whilst a positive value indicates a dosimetric advantage for one or other of the photon plans. In both plots, the change in mean dose is expressed as a percentage of the mean target dose.

From Fig. 2A,B, it can be seen that in all but one OAR of one case, it is predicted that the use of protons would reduce the mean dose by up to 80 percentage points in comparison to standard photon planning techniques and by up to 50 percentage points compared to the intensity modulated plans. In only one OAR of one case (the brain stem for case 8 ) is the mean dose reduced through the use of intensity modulated photons. This particular case is studied in more detail below.

The mean dose to which an OAR is irradiated is proportional to the integral dose delivered to that organ. As such, this measure may only have direct relevance to organs that can be considered to have a parallel (or near parallel) architecture. As a measure of 'hot spot' doses in the OARs, we have plotted in Fig. 2C,D the difference in maximum significant dose $\left(D_{\text {sig }}(\mathrm{OAR})\right)$ between the proton plan and either photon plan for each OAR. This concept is exactly analogous to the cold spot (minimum significant dose) analysis described above [11]. For OAR hot spot analysis, a volume equivalent to a sphere of radius 0.75 $\mathrm{cm}$ [18] or $5 \%$ of the OAR volume, whichever is the smallest, has been adopted.

As with the mean dose analysis, for no case are there any OARs with a reduced $D_{\text {sig }}$ resulting from standard photon planning in comparison with the proton plan. When compared to the IM photon plan, 27 of the 38 OARs (70\%) assessed over all the cases have reduced hot spot intensity through the use of protons, whereas in eight of the nine cases, the reverse is true, with the IM photon plans exhibiting reduced hot spot intensity in at least one OAR. Of these, cases 5,6,7,8 each have OARs for which the $D_{\text {sig }}$ is reduced by more than $10 \%$ of the mean target dose in comparison with the proton plan. These cases will be studied in more detail below.

Although useful parameters for quantifying integral dose and hot spot intensity for individual OARs, both the mean and maximum significant dose provide little indication of the dose-volume characteristics contained in the dose 
Table 4

Numerical identifiers of all OARs of all cases plotted in Figs. 2 and 3, together with the corresponding tolerance doses used for the IM photon plans $^{\mathrm{a}}$

\begin{tabular}{|c|c|c|c|}
\hline Case number & Oar number & Oar description & Tolerance dose $(\%)$ \\
\hline \multirow[t]{6}{*}{1} & 1 & Right eye & 20 \\
\hline & 2 & Left eye & 20 \\
\hline & 3 & Brain stem/pons & 70 \\
\hline & 4 & Whole brain & \\
\hline & 5 & Left optic nerve & \\
\hline & 6 & Right optic nerve & \\
\hline \multirow[t]{7}{*}{2} & 1 & Bladder & 65 \\
\hline & 2 & Right acetabulum & 65 \\
\hline & 3 & Rectum & 25 \\
\hline & 4 & Vagina/uterus & 30 \\
\hline & 5 & Labiae & 50 \\
\hline & 6 & Right femur & \\
\hline & 7 & Intestine & \\
\hline \multirow[t]{4}{*}{3} & 1 & Spinal cord & 55 \\
\hline & 2 & Right kidney & 35 \\
\hline & 3 & Left kidney & 35 \\
\hline & 4 & Liver & \\
\hline \multirow[t]{7}{*}{4} & 1 & Right kidney & 20 \\
\hline & 2 & Left kidney & 20 \\
\hline & 3 & Bladder & 80 \\
\hline & 4 & Total pelvis & \\
\hline & 5 & Total abdomen & \\
\hline & 6 & Vertebral body & \\
\hline & 7 & Spinal cord & \\
\hline \multirow[t]{4}{*}{5} & 1 & Rectum & 65 \\
\hline & 2 & Bladder & 85 \\
\hline & 3 & Large bowel & 85 \\
\hline & 4 & Total pelvis & \\
\hline \multirow[t]{4}{*}{6} & 1 & Brain & \\
\hline & 2 & Brain stem & 60 \\
\hline & 3 & Right eye & 10 \\
\hline & 4 & Right optic nerve & 65 \\
\hline \multirow[t]{3}{*}{7} & 1 & Spinal cord & 10 \\
\hline & 2 & Right lung & 10 \\
\hline & 3 & Left lung & 10 \\
\hline \multirow[t]{2}{*}{8} & 1 & Brain stem & 40 \\
\hline & 2 & Posterior fossa & \\
\hline 9 & 1 & Brain stem & 60 \\
\hline
\end{tabular}

${ }^{\mathrm{a}}$ The tolerance doses are expressed as a percentage of the mean target dose.

volume histograms (DVHs). In order to somewhat characterise the DVHs of the individual OARs, we have also plotted the volumes of each OAR that would receive $70 \%$ $\left(V_{70}(\mathrm{OAR})\right)$ and $50 \%\left(V_{50}(\mathrm{OAR})\right)$ of the mean target dose. In Fig. 3, these results have been plotted in a similar fashion to Fig. 2. In all plots of Fig. 3, the difference in volume is expressed as a percentage of the total volume of each OAR.

At the $70 \%$ dose level, the higher dose conformity resulting from the use of protons compared to standard photon techniques is clearly demonstrated (Fig. 3A). In only one case are the volumes of all OARs receiving $70 \%$ dose or more comparable (case 3), whilst for all other cases, the proton plans reduce $V_{70}$ in the majority of OARs by as much as $80 \%$ of the OAR volume (e.g. brain stem and femur in cases 1,2 respectively). At the $50 \%$ dose level, the pattern is similar, with a generally larger improvement through the use of protons, particularly in case 2 , where $V_{50}$ is reduced by over $90 \%$ of the total volume in two OARs (acetabulum and femur).

In contrast to the results for the standard photon plans, when comparing protons with IM photons, no general improvement at the $70 \%$ dose level is evident. The differences between the plans are generally much less, and, although the majority of OARs have a reduced $V_{70}$ with protons, in four cases $(4,5,7,8)$, some OARs show a reduction in $V_{70}$ by as much as $40 \%$ for the IM photon plan (Fig. $3 \mathrm{~B})$. At the $50 \%$ dose level, the same comparison produces results similar to those for the standard photon plans, with a reduction of $V_{50}$ for the majority of OARs through the use of protons and only one OAR of one case showing a dosimetric advantage for the IM photon plan (the brain stem in case 8).

\subsection{Selected case studies}

In Figs. 2 and 3, we have attempted to summarise both doses and irradiated volumes of OARs such that the reader can have an overview of the characteristics of the different planning methods when applied to very disparate cases. However, to gauge fully the real clinical relevance of doses to particular OARs, a more detailed analysis is essential. In this section, we have selected four cases of particular interest to be studied in more detail.

\subsubsection{Case two}

The first case we discuss in detail is of a 64-year-old female with a malignant melanoma in the right lumbar region. The target volume is located in a superficial location anterior to the bladder and right femoral head (Fig. 1a) and extends in the long axis from the upper pelvis to a third of the way down the right thigh. The calculated volume of the target is just under 1 liter $(920 \mathrm{ccm})$ and the prescribed dose was 66.6 Gy. DVHs for all defined VOIs are shown in Fig. 4. For the anatomical VOIs, where necessary, the DVHs has been calculated with those parts of the target volume that partially overlap with the VOI removed.

All the DVHs for this case show a clear and substantial separation between the proton plan and either of the standard or intensity modulated photon plans, indicating a significant dosimetric advantage in the use of protons for all aspects of this case. In particular, the DVHs for the outline, represents the dose-volume characteristics for all the non-target tissues and graphically shows the four fold reduction of the volume of normal tissue irradiated to $30 \%$ (20 Gy) or more by the proton plan (already indicated in Table 2). For the OARs, the use of protons in this case could result in a drop of up to $30 \mathrm{~Gy}(45 \%)$ in the mean dose to the acetabulum compared to the intensity modulated plan, as well as reductions in ICRU hot spot intensity of up to 23 Gy $(35 \%)$ in the case of the vagina. However, at about the $70 \%$ dose level (46 Gy) and above the irradiated volumes are similar for both the proton and IM photon plans. 

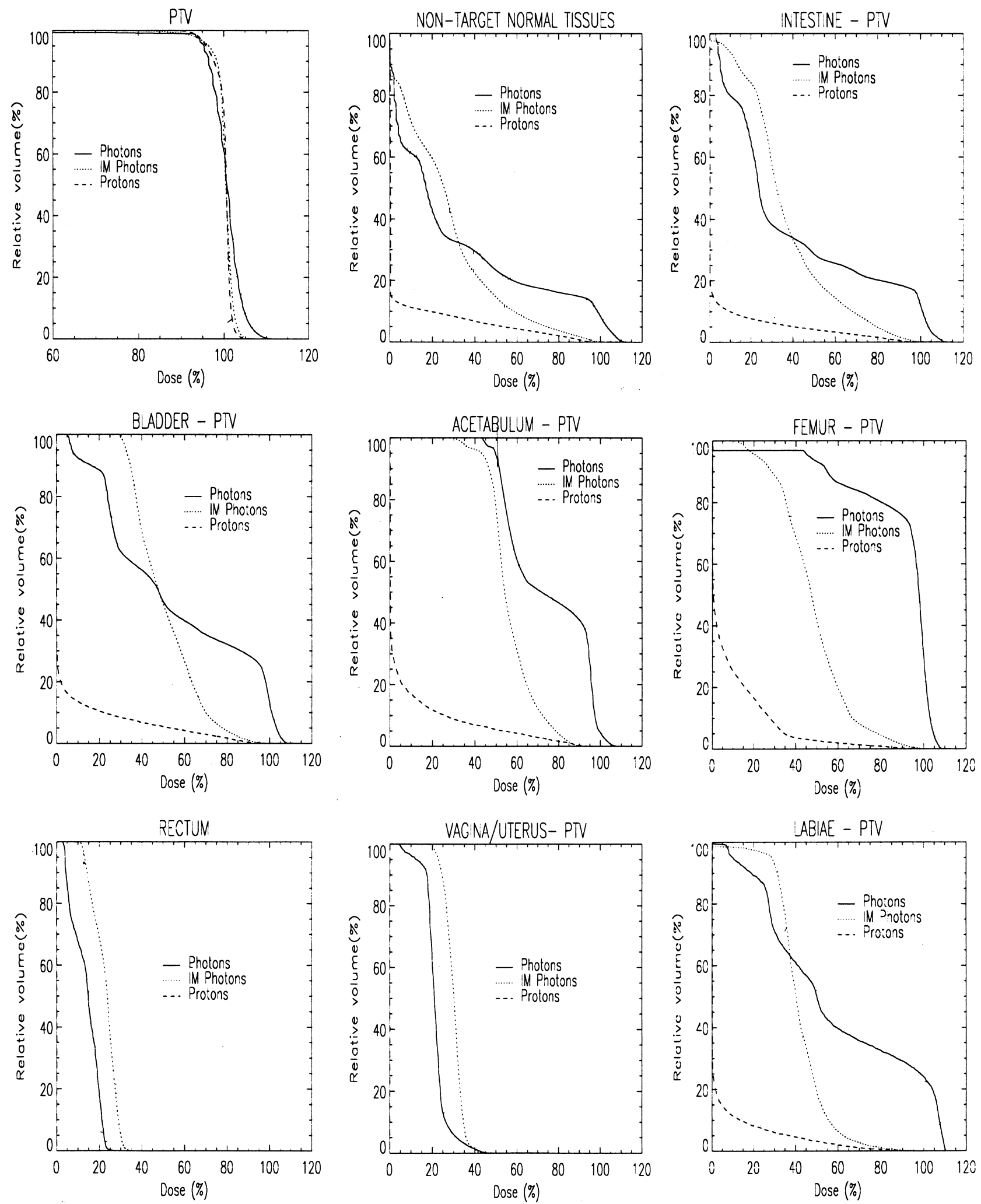

Fig. 4. Note, proton DVHs are not visible for the rectum and vagina as this plan deposits no dose in either of these organs.

\subsubsection{Case five}

This case is of a cervix carcinoma in a patient previously treated with three cycles of chemotherapy. The target volume is situated close to the bladder and rectum with both structures impressing into the defined target. As well as the target volume, VOIs of the rectum, bladder, portions of the small 

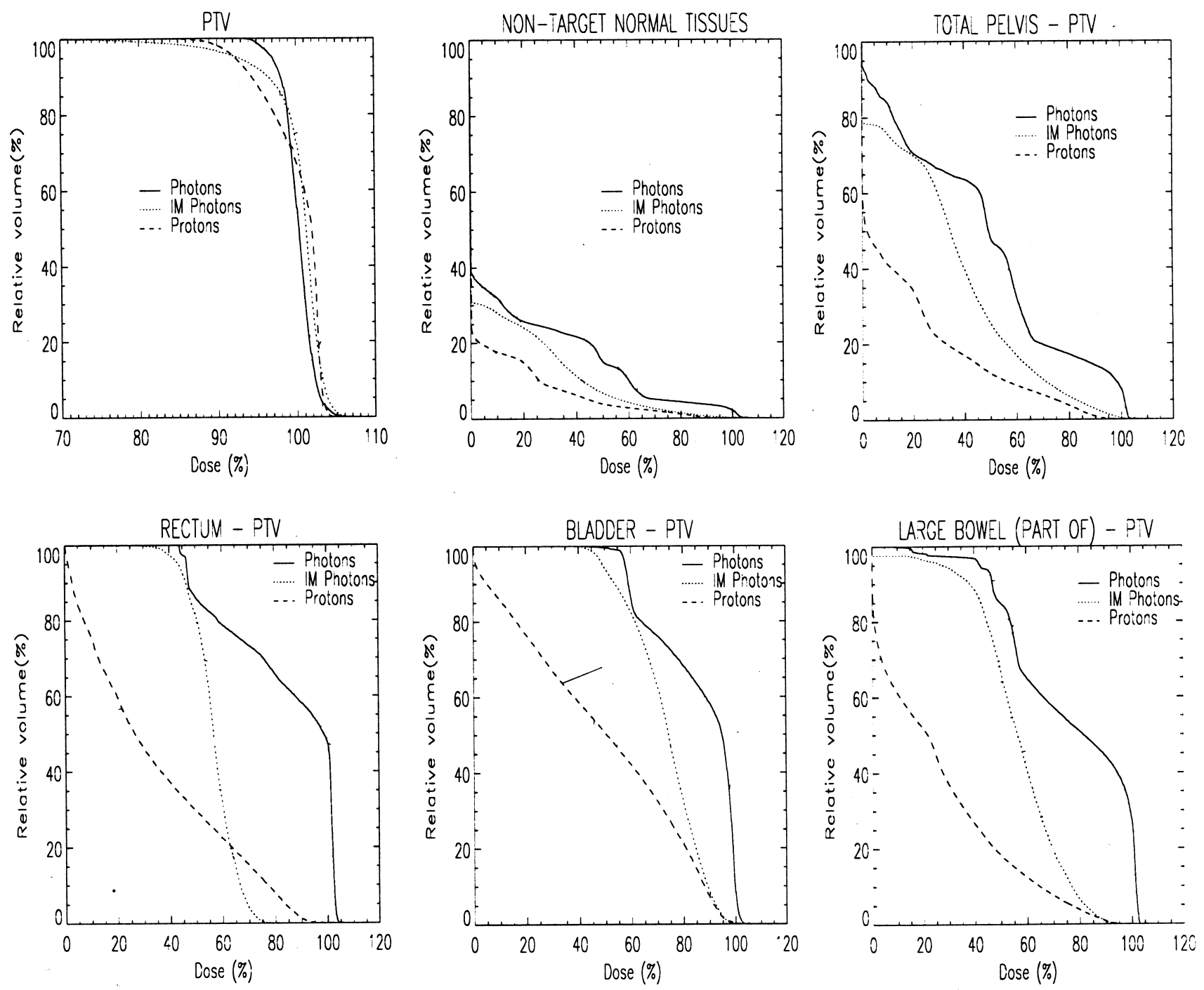

Fig. 5. DVHs for the PTV and all defined OARs for case 5.

bowel and the whole pelvic region have been defined. DVHs of all these structures are shown in Fig. 5.

For the target DVHs in Fig. 5, it can be seen that the IM photon plan has a shallower shoulder than either of the other plans, indicating some compromising of the target dose by this modality. In nearly all the OAR DVHs, a clear separation between the proton and photon (both standard and IM) plans are seen. However, perhaps the most interesting feature of this set of DVHs is the overlapping of the proton and IM DVH curves above the 60\% level for the rectum, indicating an improved high dose sparing of this organ by the IM photon plan. This overlapping is also seen in Fig. 3B as the single OAR appearing above the zero line for case 5. In Fig. 6, the proton and IM photon dose distribution are shown zoomed in the region of the target-rectum border. This figure clearly shows that in order to reduce the maximum dose to the rectum, the intensity modulated plan, has in fact, compromised the dose to the target in the immediate vicinity of the rectal surface, such as to reduce the maximum dose to that organ. This dose gradient is reflected in the larger volume of target under dosage seen in Table 2 ( $81 \%$ cold spot for the IM photon plan compared to $93 \%$ for the proton plan), as the majority of the dose less than $90 \%$ occurs along this border region of the target volume. For the proton plan, no such compromise has been made, resulting in improved target coverage but consequently to an increased dose to the rectal surface.

\subsubsection{Case seven}

This is a simulated case of a thyroid tumor, extending down into the mediastinum to the level of the mid-lung. The case was simulated on a rando phantom and VOIs for the target, both lungs and spinal cord were defined from CT slices taken of the phantom. The case was inspired by an actual case that had occurred in a clinic outside of this collaboration [13]. DVHs for all the defined VOIs are shown in Fig. 7. The target DVHs for both photon plans is clearly inferior to that of the proton plan, with substantial 


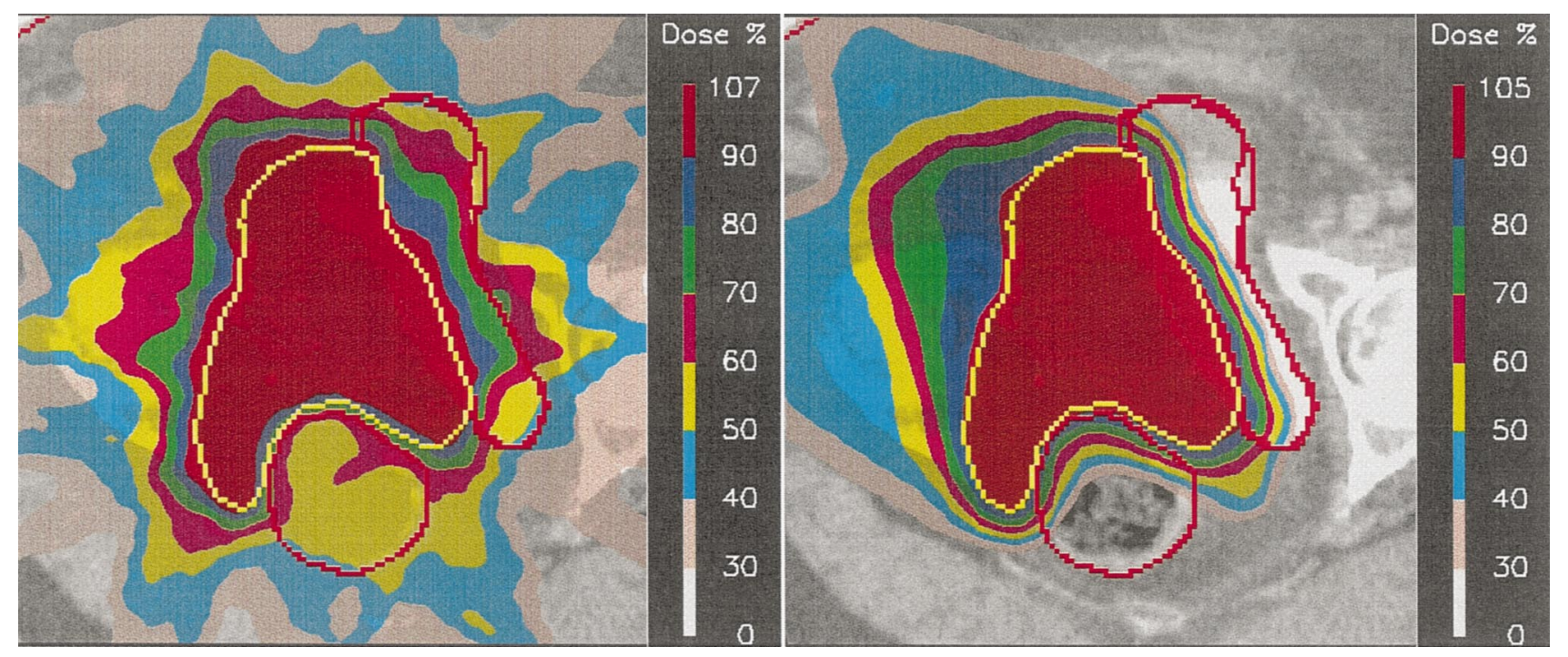

Fig. 6. Dose distributions for the IM photon (left image) and proton (right image) plan for case five, zoomed in the area of the target rectum boundary. The clearly reduced dosing to the rectal surface by the IM photon plan (between 60-80\% of the target dose compared to 80-90\% for the proton plan) has been achieved at the cost of target dose homogeneity in the neighbourhood of the rectum when compared to the proton plan.
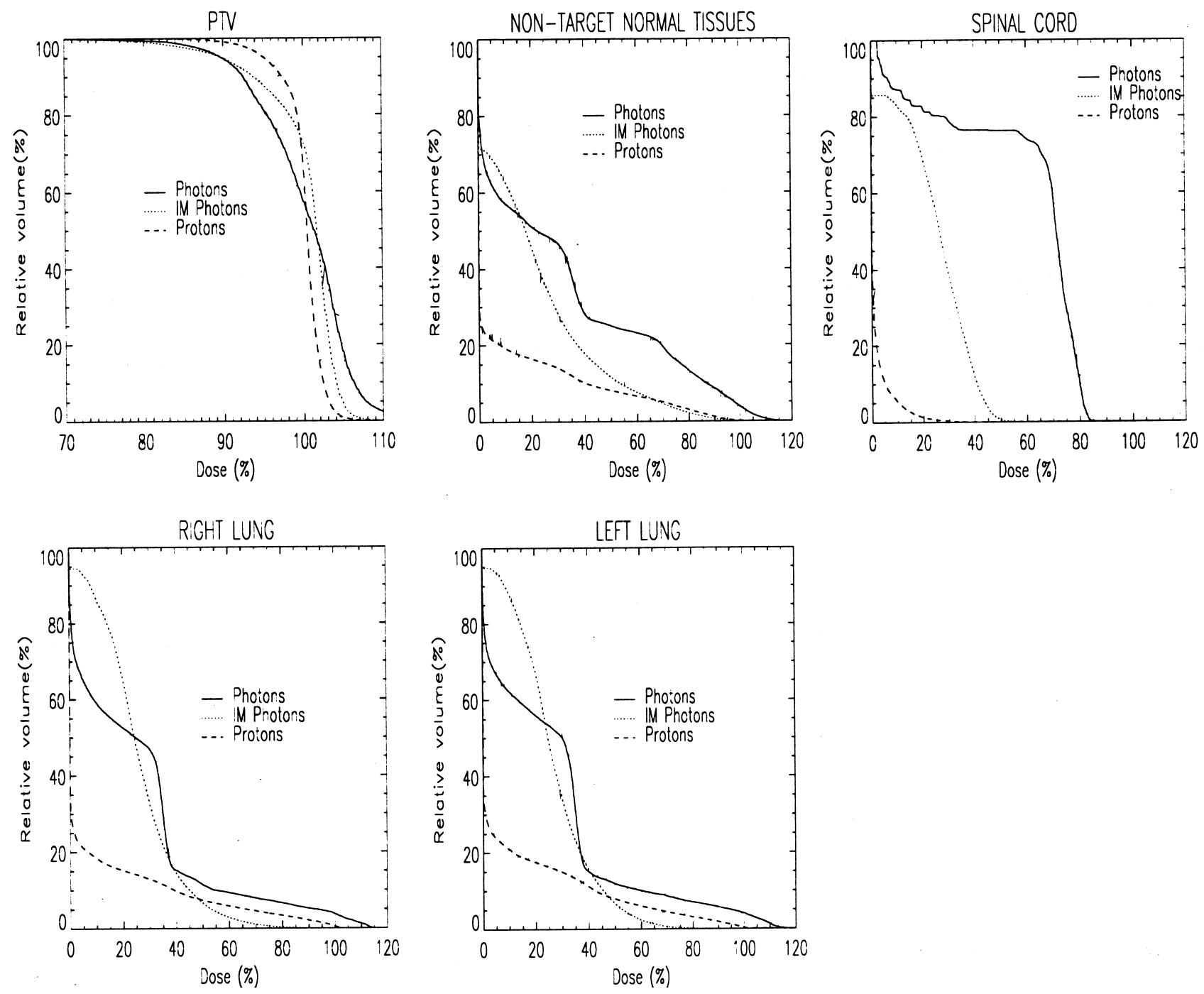

Fig. 7. DVHs for the PTV and all defined OARs for case 7. 

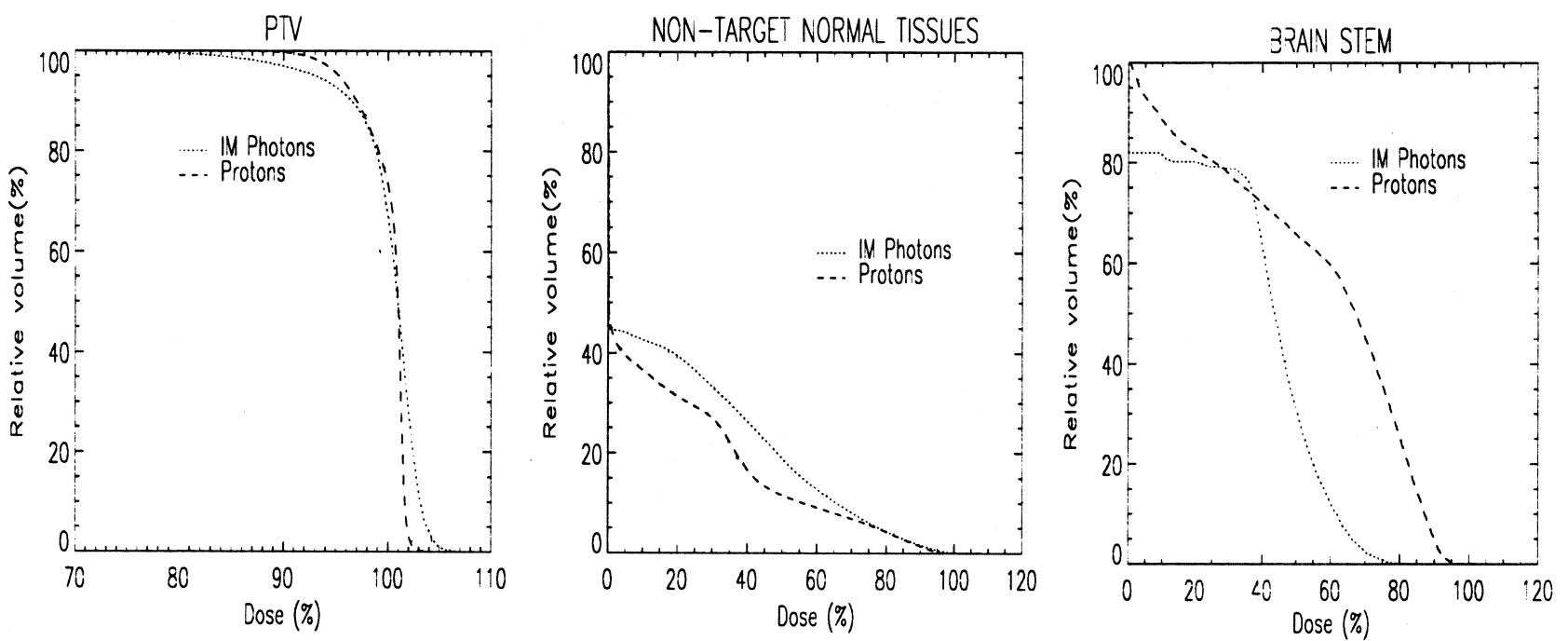

Fig. 8. DVHs for the PTV and all defined OARs for case 8.

compromising of the target dose indicated by the shallow shoulder and slow increase to the $100 \%$ volume, particularly for the IM photon plan. Again, this characteristic is reflected in the quantification provided in Table 2 .

For the OARs, the proton plan also shows a clear advantage in reducing the dose to the spinal cord (Fig. 7c). For mid to low doses, protons also considerably reduce the dose to both lungs. However, as seen in case 6 above, the crossing of the IM photon DVHs curve at the $50 \%$ dose level indicates improved high dose sparing by the use of IM photons. Again, this characteristic of the two DVHs is reflected in differences for the same OARs in Figs. 2 and 3 with all OARs being below the zero line (proton advantage) for the mean dose and the points for the two lung VOIs being above the zero line (photon advantage) for the hot spot $\left(D_{s i g}\right)$ and $V_{70}$ analysis.

\subsubsection{Case eight}

The final case is of a 45 -year-old male presenting with an acinus cell carcinoma of the right sub-lingual gland. The tumour volume was relatively large $(350 \mathrm{ccm})$ and partially wrapped around the brain stem (see Fig. 1b). DVHs for the target, non-target tissues and brain stem are shown in Fig. 8 . Only proton and intensity modulated photon plans were performed for this case. The most significant point of this case is the large separation demonstrated between the brain stem DVHs of the two plans. The IM photon plan demonstrates superior sparing of this organ at almost all dose levels, and particularly at the high $(>50 \%)$ levels. Balanced against this is a marked compromising of the dose to the target volume, clearly seen in the target DVHs and quantified somewhat in Table 2.

\section{Discussion}

In the previous sections we have presented the results of treatment planning comparisons on nine separate cases, covering a wide range of tumour types and sites. In this presentation, we have aimed to summarise as well as possible the results of each of the plans, with the aim of providing some quantitative information from which the reader can draw conclusions as to the relative merits of standard photon, intensity modulated photon and proton treatments. Here we will discuss some points that can be concluded from this work and provide pointers as to the role of the different techniques and to their future development.

Over all the cases, it can be concluded that the proton plans in general provided a better coverage of the target than either of the standard or IM photon techniques (Table 2), in addition to significantly reducing mean doses to OARs and the volumes of normal tissues and OARs irradiated to the 30 and 50\% dose levels respectively (Table 3 and Fig. 3A,B). At the $70 \%$ dose level however, little difference overall was demonstrated between the volume of OARs irradiated by the proton or IM photon plans, whilst the comparative analysis of OAR hot spots $\left(D_{\text {sig }}\right)$ showed a strong case and OAR dependency (Fig. 2B). The data presented in Figs. 2 and 3 show quantitatively what is clear qualitatively in the dose distributions of Fig. 1 that, the conformation of dose at the $70 \%$ dose level and above is essentially the same for both proton and intensity modulated photon plans, but that the lower dose 'wash' to surrounding tissues is considerably reduced through the use of protons. In drawing conclusions from these observations though, two important points should be made.

Firstly, the clinical significance of these results are a matter of some debate. It is clear that for some critical structures, it is important to reduce the dose below prescribed tolerance levels in order to reduce the possibility of side effects, and it is clear that both photon IMRT and proton therapy can reduce OAR doses, and therefore the risk of complication, in comparison to the standard photon plans used in this analysis. However, the relevance of high dose 
sparing in comparison to reduction of the mid-to-low doses depends heavily on the volume effect exhibited by each OAR. For supposed serially organised organs (such as the rectum in case 5), the comparable, and in some cases improved, sparing of some OARs at and above the tolerance dose in the IM photon plans compared to the corresponding proton plans indicates that a comparable reduction in the probability of complications to these types of organs is possible using both modalities. On the other hand, the mid-to-low dose to large volumes of the lung in case 7, or to the intestine in case 2 may restrict the effectiveness of IM photons in these particular cases in comparison with protons. However, apart from some well studied organs, such as the spinal cord and lungs, the volume effect of organs is generally not well known. A case in point is the rectum, which is generally considered to be a serial organ ( $n=0.12$ from Burman's fitting of Emami's data [10,12, 29]), but which in recent work has been suggested to be a parallel organ similar in volume response to the lungs $(n=0.9$ [37]). If this latter value were correct, then the reduced mean dose to the rectum resulting from the proton plan in case 5 (Fig. 5) would in fact be the more significant factor clinically, rather than the reduced high dose volume resulting from the IM photon plan. Clearly then, without a more accurate knowledge of the volume effect in such organs, it is very difficult to state even a relative ranking of intersecting DVHs. An interesting consequence of this uncertainty is that unless known otherwise, it is as well to reduce medium as well as high doses in all OARs as much as possible. As shown in this work, such a goal can generally be better achieved through the use of protons than through the use of either standard or IM photons. In particular, case 2 shows significantly reduced dose to all OARs through the use of protons, perhaps suggesting that large, irregularly shaped targets may be a particularly good indication for this modality.

Secondly, it is necessary to determine which differences are due to differences in radiation quality, and which are due to differences in planning technologies. Take for example case 8 . The differences in dose to the brain stem between the IM photon and proton plans are clearly significant, but are in all probability dependent not on the relative characteristics of the radiation modality used, but on the relative sophistication of the planning methods applied. In all the cases presented here, the proton plans have been calculated using a small number (maximum 4, see Table 1) of essentially homogenous fields (typically $\pm 5-10 \%$ across each field within the target volume), and in which no attempt has been made to include individual critical organs (such as the brain stem) into the optimisation process. In contrast, for the IM photon approach, the dose from a large number of different field directions (nine in total) are calculated to be in-homogenous, and are optimised using dose constraints for both targets and critical structures. In this case, the ability to define dose limits to organs at risk, coupled with the additional degrees of freedom provided by intensity modulation from multiple field directions, allows the photon opti- misation procedure to balance the dose between the target and brain stem in a way that is not possible using the proton planning techniques used in this work. A similar conclusion can be reached for the rectum in case 5 (see Figs. 5 and 6). Thus, one must be extremely cautious in concluding that photons have an advantage over protons in these cases. This is probably not an advantage of photons over protons, but is more likely an advantage of inverse planning methods over what can be considered to be the forward planning methods used for the proton plans in this study.

In almost all cases presented here, protons provide an advantage over IM photons by reducing both the mean dose and $V_{50}$ for all OARs, and it is likely that much of this reduced mid-to-low dose component stems from the advantageous physical characteristics of protons. However, a word of caution is also justified regarding this observation. It is possible that through varying the parameters used in the photon optimisations, some of the lower doses to particular OARs could be further reduced. For instance, all IM photon plans presented in this paper were performed using a dose based optimisation based on the prescription of a single tolerance dose for each OAR (see Table 4). As described by Bortfeld [4], if a dose calculation point within an OAR has a dose below this defined tolerance, then this point is assigned a zero weight and plays no further role in the optimisation (unless in subsequent iterations the dose is raised above the tolerance dose once more). With such an optimisation regime, it is clear that there is no benefit to reducing doses below the tolerance dose. Indeed, it is possible that the volume of an OAR receiving dose below tolerance is increased through the optimisation process. Such an effect can be seen clearly in the DVHs for the bladder and intestine in case 2 (Fig. 4). In each of these DVHs, the use of IMRT reduces the high dose volume delivered to these OARs in comparison to the standard photon plan, but increases the lower dose volumes.

This observation is reinforced in the analysis of case 7 (Fig. 7). Here the standard photon plan reduces the low dose volumes $(<30 \%$ of the target dose) to the lungs more than the IM photon plan. In many ways, this case is similar to a test case discussed by Wang et al. for comparing the efficacy of dose and biologically based optimisation criteria in the calculation of intensity modulated fields [49]. In that work, they found that when using a single tolerance dose optimisation, little or no improvement could be gained over standard planning techniques in the region of the lung. The results of this case reinforces this view somewhat. Wang et al. then showed that the use of biologically based cost functions had the ability to produce clinically more acceptable plans. Consequently, it is possible that for this case in particular, the use of alternative optimisation methods could result in an improved IM photon plan. Indeed, by the addition of simple dose-volume constraints into the photon optimisation code used here, it has recently been shown that the quality of plan in such circumstances can be much improved [8]. 
A final comment should be made concerning overall integral dose. It is clear from the results presented in Table 3 that the reduction of integral dose with protons is still significant, even compared to IMRT techniques (an average reduction of just over two). Although it is true that the clinical relevance of low doses to large volumes is largely unknown (except perhaps in organs with a parallel or near parallel architecture), there are cases where a reduction in overall normal tissue dose may indeed be relevant. A typical case is with paediatric patients, where it is clearly desirable to reduce the dose load to normal tissue as much as possible. However, other areas where integral dose could be important are in repeat radiotherapy treatments or perhaps in radiotherapy adjuvant to chemotherapy or surgery, in all of which there is a need to preserve the viability of normal tissues. Indeed, although the clinical relevance of integral dose may be unknown in many cases, this does not necessarily imply that integral dose is benign.

\section{Conclusions}

In this work, we have found that the use of protons in radiotherapy could potentially provide both improved target dose homogeneity and reduced mean and mid-to-low doses to critical structures when compared to either standard or IM photon treatments. Although the clinical advantage of reducing such doses is not well understood in all cases, the use of radiation modalities with superior dose localisation characteristics to that of photons remain the only way of achieving this goal and as such will inevitably play a role in the future development of radiotherapy. At the 70\% and above dose levels, the ability to spare organs at risk has been shown to be comparable for both IM photons and protons, and in three of the nine cases studied, we have demonstrated that the use of IM photons can provide significant improved high dose sparing in some critical structures. However, in each of these cases we suggest that this improvement originates from comparing inversely planned photons against forward planned protons. For a true comparison, inverse techniques must also be applied in proton therapy. Indeed, the 3D localisation of dose provided by the Bragg peak in principle provides an additional degree of freedom in which intensity can be modulated, and as such, may provide a method by which highly conformal doses can be constructed even in the most demanding of situations [28]. Consequently, a detailed comparison of inverse methods for both photons and protons is necessary to provide further insight into the relative roles of these modalities in radiation oncology.

\section{References}

[1] Bendl R, Pross J, Hoess A, Keller MA, Preiser K, Schiegel W. Virtuos - a program for virtual radiotherapy simulation and verification. In: AR Hounsell, JM Wilkinson, PC Williams, editors. XIth International
Conference on 'The use of computers in radiation therapy', Manchester, 20-24 March, 1994. pp. 226-227.

[2] Boesecke R, Bauer B, Schiegel W, Schad L, Lorenz WJ. Medical workstation for radiation therapy planning. In: Bruinvis IA, editor. IXth International Conference on 'The use of computers in radiation therapy, 1987. pp. 181-183.

[3] Bonnet DE. Current developments in proton therapy: a review. Phys. Med. Biol. 1993;38:1371-1392.

[4] Bortfeld T, Buerkelbach J, Boesecke R, Schiegel W. Methods of image reconstruction from projections applied to conformation radiotherapy. Phys. Med. Biol. 1990;35:1423-1434.

[5] Bortfeld T, Schlegel W. Optimisation of beam orientations in radiation therapy: some theoretical considerations. Phys. Med. Biol. 1993;38:291-304.

[6] Bortfeld T, Schiegel W, Rhein B. Decomposition of pencil beam kernels for fast dose calculations in three-dimensional treatment planning. Med. Phys. 1993;20:311-318.

[7] Bortfeld T, Boyer AL, Schiegel W, Kahier DL, Waldron TJ. Experimental verification of multileaf modulated conformal radiotherapy. In: Hounsell AR, Wilkinson JM, Williams PC, editors. XIth International Conference on 'The use of computers in radiation therapy', Manchester, 20-24 March, 1994, pp. 180-181.

[8] Bortfeld T, Stein J, Preiser K. Clinically relevant intensity modulation optimisation using physical criteria. In: Leavitt DD, Starkschall G, editors. XIIth International Conference on 'The use of computers in radiation therapy', Salt Lake City, 27-30 May, 1997. pp. 1-4.

[9] Brahme A, Kallmann P, Lind BK. Optimisation of proton and heavy ion therapy using an adaptive inversion algorithm. Radiother. Oncol. 1989;15:189-197.

[10] Burman C, Kutcher GJ, Emami B, Goitein M. Fitting of normal tissue data to an analytical function. Int. J. Radiat. Oncol. Biol. Phys. 1991;21:123-136.

[11] Dykstra C, Bortfeld T, Debus J, Lomax A, Harrop R, Schiegel W, Munkel G. Characterisation of dose distributions in radiation therapy plans. Radiother. Oncol. 1996;41:281-284.

[12] Emami B, Lyman J, Brown A, et al. Tolerance of normal tissue to therapeutic irradiation. Int. J. Radiat. Oncol. Biol. Phys. 1991;21:109122.

[13] Esik G, Bortfeld T, Bendel R, Nemeth G, Schiegel W. Inverse radiotherapy planning for a concave-convex PTV in cevical and upper mediastinal regions. Strahienther. Onkol. 1997;173:193-200.

[14] Goitein M, Chen GTY. Beam scanning for heavy charged particle radiotherapy. Med. Phys. 1983;10:831-840.

[15] Goitein M. The comparison of treatment plans. Sem. Radiat. Oncol. 1992;2:246-256.

[16] Gokhale P, Hussein EMA, Kuikani N. Determination of beam orientation in radiotherapy planning. Med. Phys. 1994;21:393-400.

[17] Holmes TW, Mackie TR, Reckwerdt PI, Deasy JO. A prototype inverse treatment planning algorithm for tomotherapy. In: Hounsell AR, Wilkinson JM, Williams PC, editors. XIth International Conference on 'The use of computers in radiation therapy', Manchester, 2024 March, 1994. pp. 62-63.

[18] ICRU-50, Prescribing, recording and reporting photon beam therapy. Bethesda, MD, 1993.

[19] Iain NL, Kahn MG, Graham MV, Purdy JA. 3D conformal radiation therapy: V. Decision-Theoretic evaluation of radiation treatment plans. In: Hounsell AR, Wilkinson JM, Williams PC, editors. XIth International Conference on 'The use of computers in radiation therapy', Manchester, 20-24 March, 1994. pp. 8-9.

[20] Kallmann P, Lind BK, Brahme A. An algorithm for maximising the probability of complication free tumour control in radiation therapy. Phys. Med. Biol. 1992;37:871-890.

[21] Kanai T, Kanai K, Kumamoto Y, Ogawa H, Yamada T, Matsuzawa H. Spot scanning system for radiotherapy. Med. Phys. 1980;7:365369.

[22] Koehler AM, Schneider RJ, Sisterson JM. Range modulators for protons and heavy ions. Nucl. Instrum. Methods 1975;131:437-440. 
[23] Koehler AM, Schneider RJ, Sisterson JM. Flattening of proton dose distributions for large field radiotherapy. Med. Phys. 1977;4:297-301.

[24] Lee M, Wynne C, Webb S, Nahum AE, Dearneley DA. Comparison of proton and mega-voltage $\mathrm{X}$-ray treatment planning for prostate cancer. Radiother. Oncol. 1994;33:239-253.

[25] Ling CC, Burman C, Chui C, Kutcher GJ, Leibel SA, LoSasso T, Mohan R, Bortfeld T, Reinstein L, Spirou S, Wang XH, Wu Q, Zelefsky M, Fuks Z. Conformal radiation treatment of prostate cancer using inversely-planned intensity-modulated photon beams produced with dynamic multileaf coliimation. Int. J. Radiat. Oncol. Biol. Phys. 1996;35:721-730.

[26] Lomax A, Scheib S, Munkel G, Blattmann H. The comparison of spot scanning proton radiotherapy with conventional photon therapies. In: Hounsell AR, Wllkinson JM, Williams PC, editors. XIth International Conference on 'The use of computers in radiation therapy', Manchester, 20-24 March, 1994. pp. 366-367.

[27] Lomax AJ, Pedroni E, Schaffher B, Scheib S, Schneider U, Tourovsky A. 3D treatment planning for conformal proton therapy by spot scanning. In: Faulkner K et al., editors. Proc. 19th L H Gray Conference, London: BIR publishing, 1999. pp. 67-71.

[28] Lomax A. Intensity modulation for proton radiotherapy, Phys. Med. Biol. 1999;44:185-205.

[29] Lyman JT, Wolbarst B. Optimisation of radiation therapy III: A method of assessing complication probabilities from dose-volume histograms. Int. J. Radiat. Oncol. Biol. Phys. 1987;13:103-109.

[30] Mageras GS, Mohan R. Application of fast simulated annealing to optimisation of conformal radiation treatments. Med. Phys. 1993;20:639-647.

[31] Miralbell R, Croweli C, Suit HD. Potential improvement of three dimensional treatment planning and proton therapy in the outcome of maxillary sinus cancer. Int. J. Radiat. Oncol. Biol. Phys. 1991;22:305-310.

[32] Miralbell R, Urie MM. Potential improvement of three dimensional treatment planning and proton beams in fractionated radiotherapy of large cerebral arteriovenous malformations. Int. J. Radiat. Oncol. Biol. Phys. 1993;25:353-358.

[33] Miralbell R, Lomax A, Borfeld T, Rouzaud M, Carrie C. Potential role of proton therapy in the treatment of paediatric medulloblastoma/ primitive neuroectodermal tumors: reduction of the supratentorial target volume. Int. J. Radiat. Biol. Phys. 1997;38:477-484.

[34] Miralbell R, Lomax A, Russo M. Potential role of proton therapy in the treatment of pediatric medulloblastoma/primitive neuro-ectodermal tumors: spinal theca irradiation. Int. J. Radiat. Biol. Phys. 1997;38:805-811.

[35] Mohan R, Mageras GS, Baldwin B, et al. Clinically relevant optimisation of 3D conformal treatments. Med. Phys. 1992;19:933-944.
[36] Munzenrider JE, Brown AP, Chu JCH, et al. Numerical scoring of treatment plans. Int. J. Radiat. Oncol. Biol. Phys 1991;21:147-163.

[37] Nahum AE. The potential of normal-tissue radiobiology for the physics of conformal therapy. In: Nahum A, Peacock J, editors. Tissue effects in radiotherapy: physics meets biology, Betchworth, UK, 1416 July, 1997.

[38] Niemierko A. Random search algorithm (RONSC) for optimisation of radiation therapy with both physical and biological end points and constraints. Int. J. Radiat. Oncol. Biol. Phys. 1992;23:89-98.

[39] Niemierko A, Urie M, Goitein M. Optimisation of 3D radiation therapy with both physical and biological end points and constraints. Int. J. Radiat. Oncol. Biol. Phys. 1992;23:99-108.

[40] Pedroni E, Bacher R, Blattmann H, et al. The $200 \mathrm{MeV}$ proton therapy project at PSI: conceptual design and practical realisation. Med. Phys. 1995;22:37-53.

[41] Preiser K, Bortfeld T, Hartwig K, Schlegel W, Stein J. A new program for inverse radiotherapy planning. In: Leavitt DD, Starkschall G, editors. XIIth International Conference on 'The use of computers in radiation therapy', Salt Lake City, 27-30 May, 1997. pp. 425-428.

[42] Sherouse GW. Dose homogenisation using vector analysis of dose gradients. In: Hounsell AR, Wilkinson JM, Williams PC, editors. XIth International Conference on 'The use of computers in radiation therapy', Manchester, 20-24 March, 1994. pp. 248-249.

[43] Scheib S, Pedroni E. Dose calculations and optimisation for 3D conformal voxel scanning. Radiat. Environ. Biophys. 1996;31:251256.

[44] Sisterson J. Particles Newsletter 21 (Harvard cyclotron laboratory), January 1998.

[45] Slater JD, Slater JM, Wahien S. The potential for proton therapy in locally advanced carcinoma of the cervix. Int. J. Radiat. Oncol. Biol. Phys. 1992;22:343-348.

[46] Tatsuzaki H, Urie MM, Lingwood R. Comparative treatment planning: proton versus $\mathrm{X}$-ray beams against glioblastoma multiforme. Int. J. Radiat. Oncol. Biol. Phys. 1992;22:265-274.

[47] Tatsuzaki H, Urie MM, Willet CG. 3D comparative study of proton versus. X-ray radiation therapy for rectal cancer. Int. J. Radiat. Oncol. Biol. Phys. 1992;22:369-374.

[48] Urie MM, Goitein M. Variable versus fixed modulation of proton beams for treatments in the cranium. Med. Phys. 1989;16:16.

[49] Wang X, Mohan R, Jackson A, Leibel SA, Fuks Z, Ling CC. Optimisation of intensity modulated 3D treatment plans based on biological indices. Radiother. Oncol. 1995;37:140-152.

[50] Webb S. Optimisation of conformal radiotherapy dose distributions by simulated annealing. Phys. Med. Biol. 1989;34:1349-1370.

[51] Wilson RR. Radiological use of fast protons. Radiology 1946;47:487491. 